Case Report

\title{
Recurrence of Solid Pseudopapillary Tumor: A Rare Pancreatic Tumor
}

\author{
Chandra Punch, Nupur Garg, and Penelope Harris \\ Madigan Army Medical Center, Tacoma, WA, USA \\ Correspondence should be addressed to Chandra Punch; chandra.s.punch.mil@mail.mil
}

Received 21 July 2016; Accepted 3 November 2016

Academic Editor: Constantine Gennatas

Copyright (C) 2016 Chandra Punch et al. This is an open access article distributed under the Creative Commons Attribution License, which permits unrestricted use, distribution, and reproduction in any medium, provided the original work is properly cited.

Solid pseudopapillary tumor of the pancreas (SPTP) is a rare disease of young females that does not usually recur after resection. Here we report a case of an elderly female with history of SPTP ten years ago who presented with anorexia and a palpable left lower quadrant abdominal mass. Imaging revealed metastatic disease and US-guided biopsy of the liver confirmed the diagnosis of SPTP. Due to her advanced age and comorbidities, she elected to undergo hospice care. The objective of this case report is to increase awareness of this tumor and its possibility of recurrence, necessitating further guidelines for follow-up.

\section{Introduction}

Solid pseudopapillary tumor of the pancreas (SPTP) is a rare pancreatic neoplasm described as a benign entity with occurrence most commonly seen in younger females (average age of 24 years) [1]. Most cases are diagnosed as an incidentally noted large mass; however when symptomatic it usually causes abdominal pain. The tumor is generally indolent in nature and survival following primary resection approaches $95 \%$ at 5 years with recurrence rates of $6.2 \%$ reported in case reports [2]. We describe a unique case of SPTP in an elderly female with recurrence many years after her primary diagnosis.

\section{Case}

An 88-year-old Caucasian female with history of SPTP diagnosed and resected 10 years prior to pathology revealing Ki-67 index of 2-3\% presented with complaints of anorexia, dry heaving, increased somnolence, and a palpable left lower quadrant abdominal mass. Imaging revealed scattered soft tissue masses throughout the abdominal mesentery as well as multiple hypodense, heterogeneously enhancing lesions within the liver (Figure 1), consistent with metastases and similar to her imaging findings at diagnosis. US-guided biopsy of the liver confirmed the diagnosis of metastatic SPTP, staining positively for vimentin, CD10, and neuronspecific enolases (Figure 2). Due to her advanced age and comorbidities, she was a poor surgical candidate and elected best supportive care. She was transferred to inpatient hospice and passed away approximately a month after her initial presentation.

\section{Discussion}

Solid pseudopapillary tumor of the pancreas was first described by Dr. Virginia Frantz in 1959 and later the World Health Organization (WHO) in 1996 defined the tumor as a low grade malignant neoplasm of the exocrine pancreas [1]. The tumor currently represents only $1-2 \%$ of exocrine pancreatic tumors and has rarely been recorded in elderly females, such as our patient, initially diagnosed at 78 years old. Often an incidental finding, it can be associated with abdominal discomfort/pain, a palpable mass, anorexia, nausea, vomiting, and weight loss [1]. The etiology of SPTP is unknown; however it has been postulated to derive from either primitive pancreatic cells or cell lines of the female genital bud [3]. Imaging (US, CT/MRI) is the primary diagnostic tool and will reveal a solid and cystic mass with irregular calcifications seen in $20 \%$ of cases [4]. Definitive diagnosis is confirmed with needle biopsy. Cytologic analysis is diagnostic in $75 \%$ of cases with positive vimentin, CD10, and beta-catenin stains differentiating SPTP from pancreatic neuroendocrine tumors. Histology will often reveal branching papillae with myxoid stroma [5]. This tumor has been reported to reach 


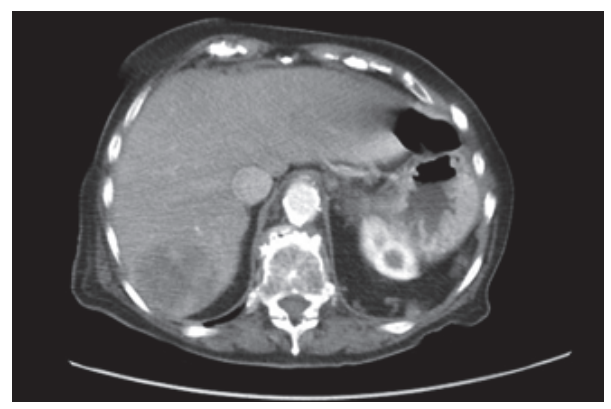

FIgURE 1: Axial view of abdominal CT scan with a hypodense mass in the liver measuring $50 * 55 \mathrm{~mm}$ in size.
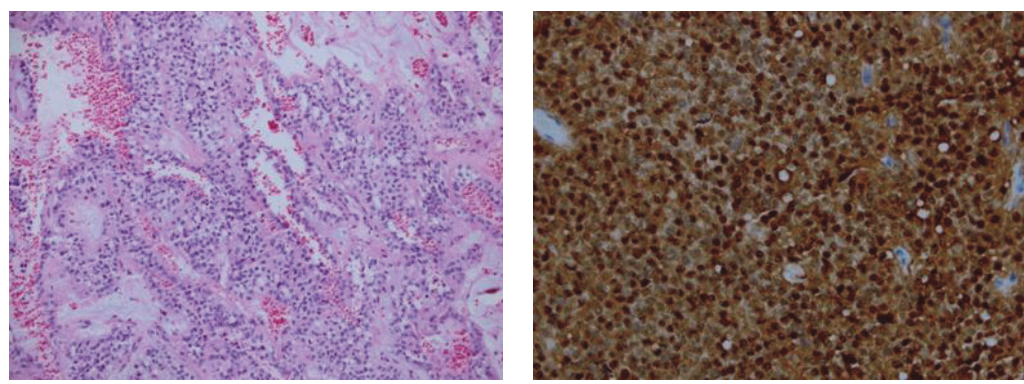

FIGURE 2: Pathology of solid pseudopapillary tumor of the pancreas $\mathrm{H}$ and $\mathrm{E}$ stain and B-catenin stain with the prominent nuclear staining.

up to $18 \mathrm{~cm}$ and even then is generally thought to be of low malignant potential and overall excellent prognosis. Malignant cases are defined as solid pseudopapillary carcinomas and are treated with resection [6]. Metastases are seen in 10$15 \%$ of advanced cases, with a tendency to localize to regional lymph nodes, liver, and peritoneum/omentum. Additional treatments, including chemotherapy and radiotherapy, have been noted in some cases with good response but are in general not used, and the 5-year survival rate after resection approaches 95\% [7].

\section{Conclusion}

While previously described as a tumor of young females, solid pseudopapillary tumor of the pancreas (SPTP) can occur in advanced age. Although the five-year survival rate following resection approaches 95\%, tumor recurrence has been reported, potentiating a role for follow-up guidelines.

\section{Disclosure}

The views expressed are those of the authors and do not reflect the official policy of the Department of the Army, the Department of Defense, or the US Government.

\section{Competing Interests}

The authors declare that there is no conflict of interests regarding the publication of this paper.

\section{References}

[1] N. Vassos, A. Agaimy, P. Klein, W. Hohenberger, and R. S. Croner, "Solid-pseudopapillary neoplasm (SPN) of the pancreas: case series and literature review on an enigmatic entity," International Journal of Clinical and Experimental Pathology, vol. 6, no. 6, pp. 1051-1059, 2013.

[2] C. Vollmer, E. Dixon, and D. Grant, "Management of a solid pseudopapillary tumor of the pancreas with liver metastases," HPB Surgery, vol. 5, no. 4, pp. 264-267, 2003.

[3] K. Goudarzipour, A. Jenabzadeh, H. Mirzaei, B. Behnam, and H. Tavassol, "Solid pseudo-papillary tumor of the pancreas in a 10 year old girl," Iranian journal of Pediatric Hematology Oncology, vol. 5, no. 3, p. 167, 2015.

[4] M. H. Yu, J. Y. Lee, M. A. Kim et al., "MR imaging features of small solid pseudopapillary tumors: retrospective differentiation from other small solid pancreatic tumors," American Journal of Roentgenology, vol. 195, no. 6, pp. 1324-1332, 2010.

[5] S. Springer, Y. Wang, M. Dal Molin et al., "A combination of molecular markers and clinical features improve the classification of pancreatic cysts," Gastroenterology, vol. 149, no. 6, pp. 1501-1510, 2015.

[6] L. Romics Jr., A. Oláh, T. Belágyi, N. Hajdú, P. Gyurus, and V. Ruszinkó, "Solid pseudopapillary neoplasm of the pancreasproposed algorithms for diagnosis and surgical treatment," Langenbeck's Archives of Surgery, vol. 395, no. 6, pp. 747-755, 2010.

[7] N. Guo, Q. B. Zhou, R. F. Chen et al., "Diagnosis and surgical treatment of solid pseudopapillary neoplasm of the pancreas: analysis of 24 cases," Canadian Journal of Surgery, vol. 54, no. 6, pp. 368-374, 2011. 


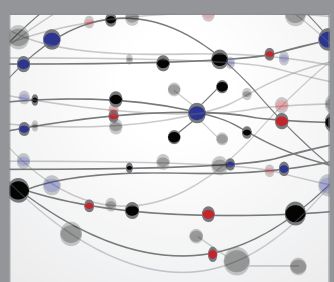

The Scientific World Journal
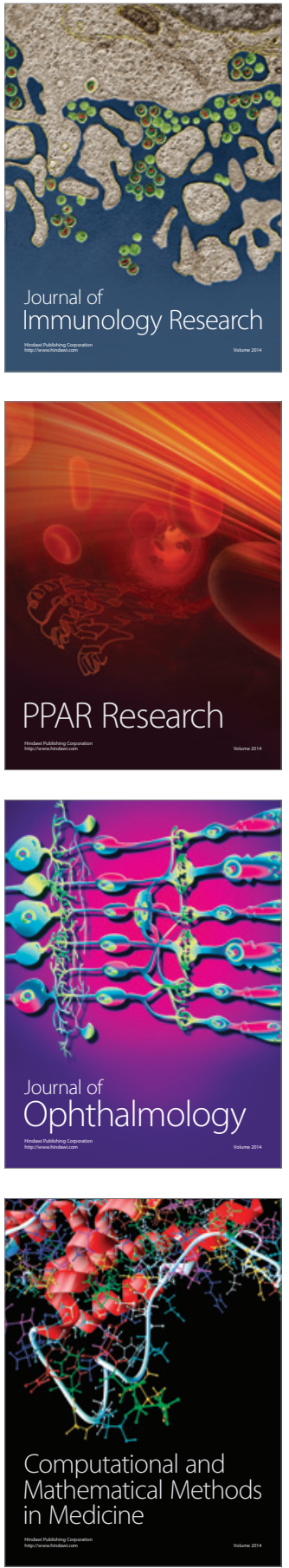

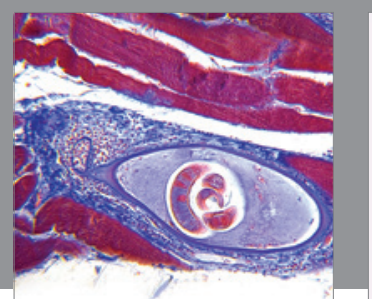

Gastroenterology Research and Practice

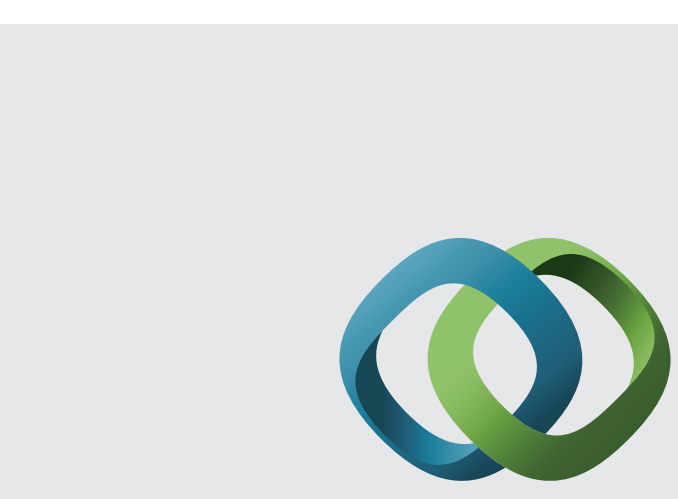

\section{Hindawi}

Submit your manuscripts at

http://www.hindawi.com
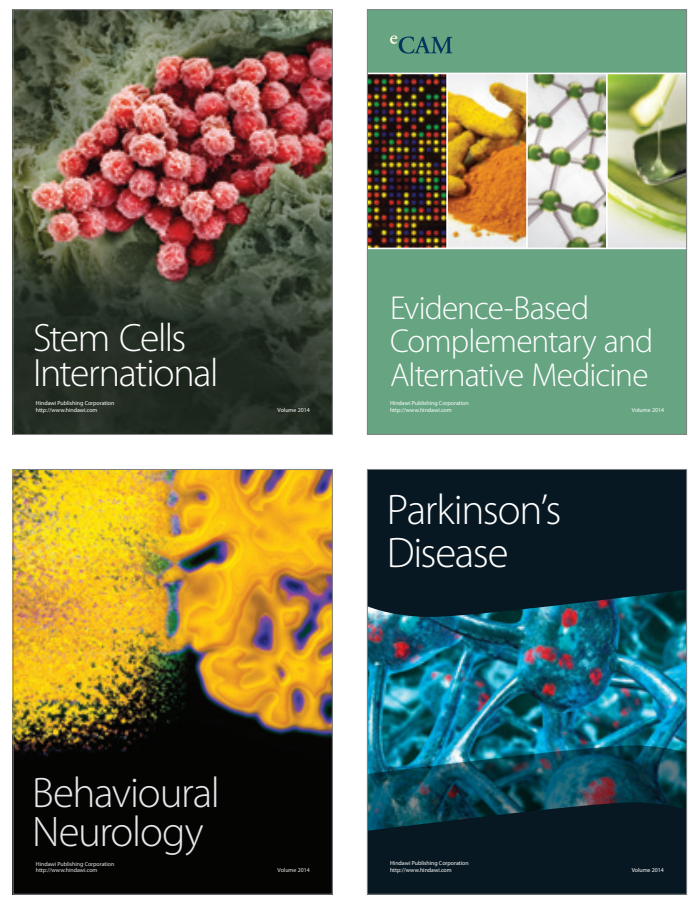
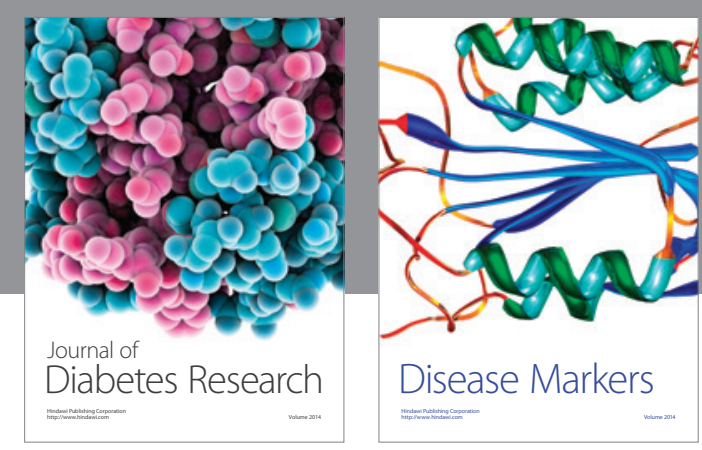

Disease Markers
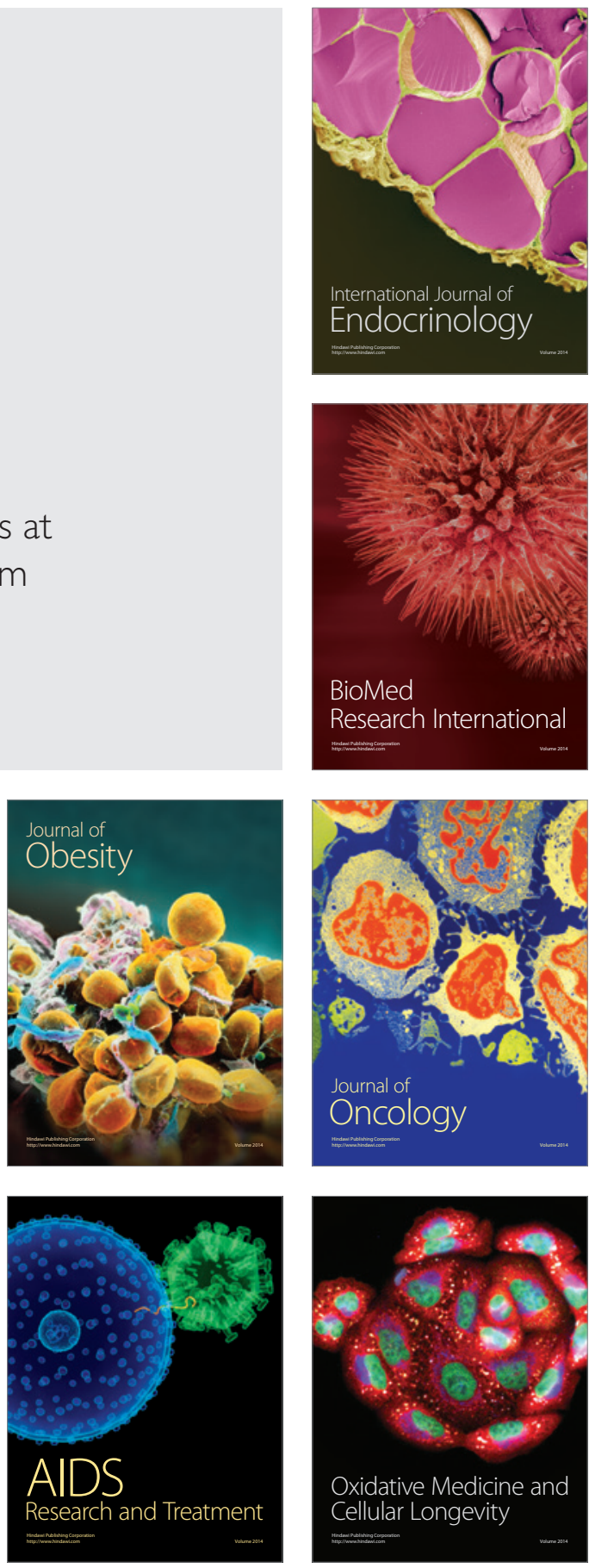